\title{
La (dés)illusion communautaire. De l'ambivalence de la notion de « communauté » en Amazonie brésilienne
}

A communitarian (des)illusion: about the notion of « community » in the Brazilian Amazon

A (des)ilusão comunitária: sobre o conceito de « comunidade » na Amazônia brasileira

\section{Émilie Stoll et Ricardo Theophilo Folhes}

\section{OpenEdition \\ Journals}

Édition électronique

URL : https://journals.openedition.org/jsa/14024

DOI : 10.4000/jsa. 14024

ISSN : 1957-7842

\section{Éditeur}

Société des américanistes

Édition imprimée

Date de publication : 31 décembre 2014

Pagination : 73-103

ISSN : 0037-9174

Référence électronique

Émilie Stoll et Ricardo Theophilo Folhes, «La (dés)illusion communautaire. De l'ambivalence de la notion de " communauté » en Amazonie brésilienne », Journal de la Société des américanistes [En ligne], 100-2 | 2014, mis en ligne le 01 janvier 2016, consulté le 04 septembre 2022. URL : http:// journals.openedition.org/jsa/14024; DOI : https://doi.org/10.4000/jsa.14024 


\title{
LA (DÉS)ILLUSION COMMUNAUTAIRE. DE L'AMBIVALENCE DE LA NOTION DE « COMMUNAUTÉ » EN AMAZONIE BRÉSILIENNE
}

\author{
Émilie STOLL et Ricardo THEOPHILO FOLHES*
}

\begin{abstract}
Cet article analyse les dynamiques et les logiques à l'œuvre dans la genèse de villages riverains d'Amazonie, appelés " communautés » depuis la théologie de la libération dans les années 1960. À partir des années 1970, l'État brésilien a entrepris leur régularisation foncière, produisant - notamment dans l'Arapiuns étudié ici - des effets de télescopage en raison du contraste entre sa propre logique institutionnelle et les pratiques locales d'occupation et de transmission de la terre. L'organisation sociale locale en « fratries résidentielles » (groupes de collatéraux et leurs conjoints habitant un même territoire) et les mécanismes d'exclusion permettant sa reproduction ont trouvé leurs limites dans une structure légale ressentie comme coercitive. Les conflits récents ayant surgi entre groupes résidentiels et, parfois, entre membres d'une même famille peuvent être interprétés dans le cadre d'une crise de représentation plus large, opérant aussi bien au niveau collectif de la « communauté », qu'à celui des choix identitaires individuels des habitants de la région. [Mots-clés : Amazonie brésilienne, communauté, caboclos, folk studies, réforme agraire, fratries résidentielles, conflits.]
\end{abstract}

A (des) ilusão comunitária: sobre o conceito de « comunidade » na Amazônia brasileira. Neste artigo abordamos as dinâmicas e as lógicas de formação de povoados - chamados de «comunidades » desde os anos 1960 pela teologia da libertação -, assim como sua regularização fundiária posterior pela União - a partir dos anos 1970 - na região do rio Arapiuns (Amazônia brasileira, Pará) e sua coincidência ou contraste com as práticas locais de ocupação e de transmissão da terra. A organização social em " fratrias residenciais » (grupos de colaterais e seus cônjuges num só território) e os mecanismos de exclusão que lhes são próprios, se veem aqui limitados por uma estrutura fundiária legal percebida como coercitiva. Os conflitos recentes entre diferentes grupos residenciais e por vezes entre membros de uma mesma família devem ser entendidos no contexto de uma crise de representação mais ampla, tanto num âmbito coletivo (das " comunidades »), quanto no que se refere às escolhas identitárias

* Département d'anthropologie de l'Université d'Aberdeen, Grande-Bretagne[em_di_sto@yahoo. com]; Institut des hautes études de l'Amérique latine, 28, rue Saint-Guillaume, 75007 Paris, France/Universidade FederaldoPará [rfolhes@gmail.com].

Journal de la Société des américanistes, 2014, 100-2, pp. 73-103. C Société des américanistes. 
individuais dos ribeirinhos desta região. [Palavras-chave: Amazônia brasileira, comunidade, caboclos, folk studies, reforma agrária, fratrias residencias, conflitos.]

A communitarian (des)illusion: about the notion of "community" in the Brazilian Amazon. In the Brazilian Amazon, the formation of rural « communities » was largely driven by the Catholic Church, and later strengthened by several external institutional actors. Now regarded as the political unit by external actors, it is the privileged interlocutor of many concerned with public policies land tenure and the redistribution of resources. In this article, using the case study of a set of rural communities along the Arapiuns River, in the state of Pará (Brazil), we show the dynamics driven in the genesis of communities' formation and later in the deliverance of land records by the State, their nesting with local practices of land use and transfer. Social organization based on " residential sibling groups ", and mechanisms of exclusion necessary to their reproduction, have found their limits in a legal structure seen as a cyclical confinement. Recent conflicts between residential groups and within families must be understood in the light of a broader crisis of representation and identity of the community. [Key words: Brazilian Amazon, community, caboclos, folk studies, agrarian reform, residential sibling group, land conflicts.]

Au Brésil, le terme « communauté » est entré dans le langage courant pour désigner de petites localités rurales non constitutives d'une unité administrative, formées en habitat groupé et organisées autour d'institutions politico-religieuses. À défaut de disposer d'un statut administratif, les communautés sont néanmoins considérées par l'administration brésilienne et les autres acteurs extérieurs comme des unités politiques locales pour la réalisation de projets publics et privés (école, réseau électrique, etc.). La communauté et ses déclinaisons sont désormais en vogue, particulièrement dans les sphères alternatives et le tiers secteur, comme en témoigne le nombre croissant de projets à vocation communautaire : tourisme, jardins, réseaux et autres communautés alternatives. Tous partagent une certaine idée du vivre-ensemble issue de représentations selon lesquelles la communauté est fondée sur la solidarité entre pairs et est imaginée comme alternative au modèle capitaliste. Ce glissement idéologique, initié dans les années 1960 par l'Église catholique (Araújo 2009), est fondé sur le principe du partage équitable du travail et des biens entre ses membres.

Entre 2008 et $2012^{1}$, nous avons étudié les dynamiques sociales à l'œuvre dans des villages de terre ferme de l'interfluve Arapiuns-Amazone, dans la commune de Santarém, dans l'ouest de l'État du Pará, au Brésil (Figure 1).

Ces villages abritent des populations rurales métisses, généralement appelées " caboclos » par la littérature scientifique (Galvão 1976 [1955] ; Nugent 1993 ; Adams et al. 2006) ou " populations traditionnelles ${ }^{2}$ » par les institutions de l'État (Araújo 2009). Les communautés riveraines de l'Arapiuns sont de taille variable, comptant entre une dizaine et une centaine de familles. Représentatives du peuplement de l'écosystème de terre ferme dans la vallée du Tapajós, elles sont 

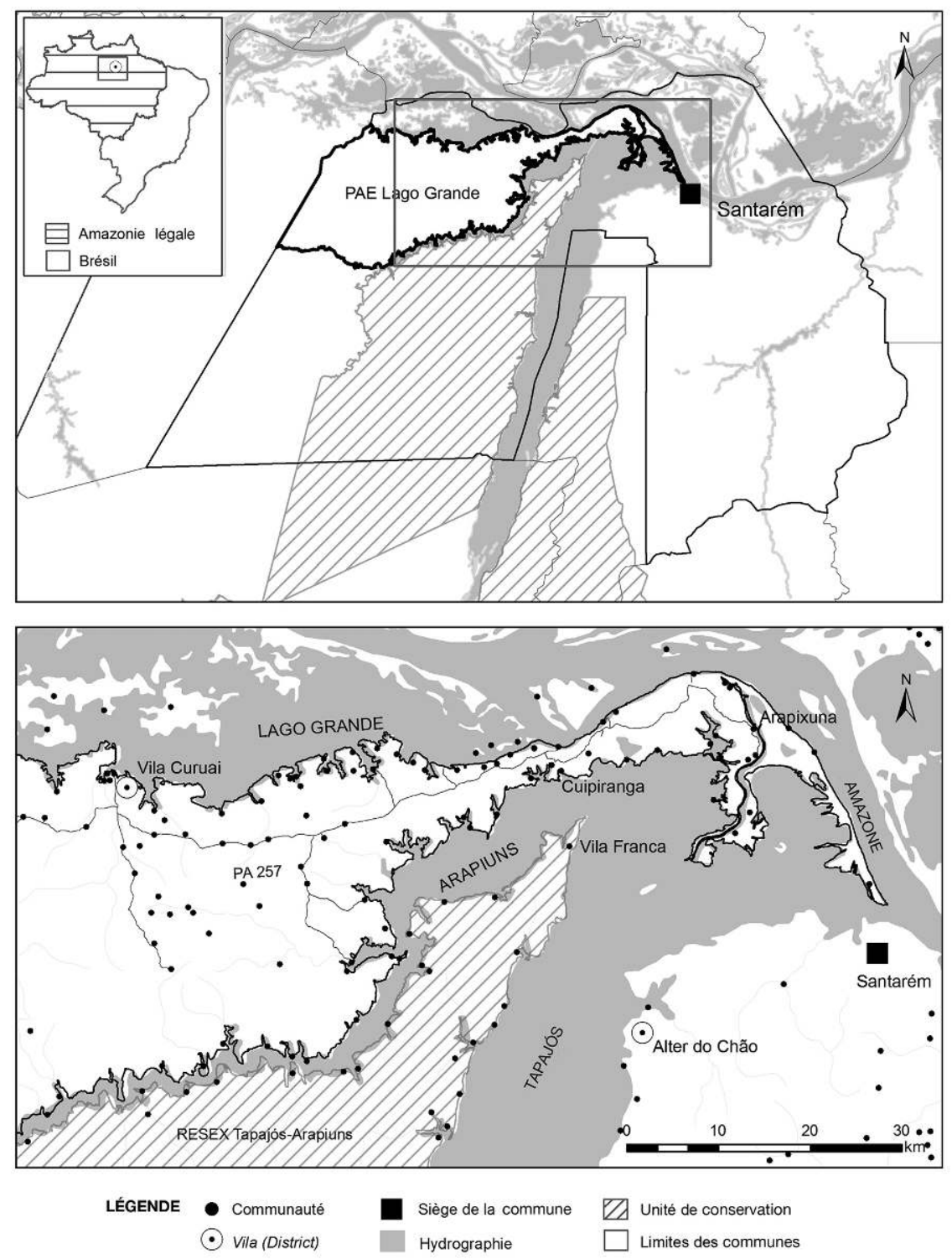

FIG. 1 - Localisation du terrain. 
par ailleurs insérées dans un paysage régional diversifié, composé de centaines de communautés situées pour une bonne part dans la várzea (écosystème très fertile de terres inondables localisées le long de l'Amazone ${ }^{3}$ ). Ces dernières présentent un modèle d'occupation et de transmission du sol assez différent de celui de la terre ferme, tel qu'observé le long de l'Arapiuns (sur la várzea, voir Nugent 1993 ; Harris 2000, 2004 ; Lima 2004; O’Dwyer 2005 ; Adams et al. 2006 ; Futemma 2006). Ceci explique pourquoi, malgré une apparente similarité dans la trajectoire de formation des villages, les enjeux, les logiques sociales et le type de conflits qu'on y observe diffèrent sur de nombreux points.

À la lumière de cet exemple amazonien, nous proposons de débattre sur la genèse des signifiants liés à la notion de communauté. L'analyse critique de ce concept sera enrichie d'une réflexion sur les logiques sous-jacentes implicitement en jeu lorsque sont évoqués les conflits communautaires. Dans un premier temps, nous présentons une description contextualisée des dynamiques d'occupation territoriale de l'Arapiuns, puis de l'inflexion des années 1960-1970 ayant mené à la formation de villages. L'arrivée des théologiens de la libération, bientôt suivie de celle des institutions de l'État, a parachevé l'implantation de communautés stables et enregistrées légalement sur le cadastre des terres fédérales. Nous montrons que le territoire était traditionnellement occupé par des groupes de collatéraux et leurs conjoints, les « fratries résidentielles », dont la perpétuation et le mode de transmission patrimoniale reposaient et reposent toujours sur l'exclusion d'une partie des descendants. La récente organisation de l'espace en communautés et la mise en œuvre par le Gouvernement, dès les années 1970, d'un vaste chantier de régularisation foncière en Amazonie se sont alors heurtées aux pratiques locales d'usage et de transmission du sol, provoquant, déjà à l'époque, des conflits entre pairs au sein des villages. Les récentes tentatives d'implantation d'une unité territoriale à usufruit collectif et les conflits qui s'en suivirent montrent bien qu'aujourd'hui encore les prises de décision politiques et économiques sont toujours réalisées selon la logique des fratries résidentielles, et non par la communauté en tant qu'unité sociale fonctionnelle, comme le suggèrent les apparences et les discours militants.

\section{LA FORMATION DES COMMUNAUTÉS DE TERRE FERME DE L'AraPIUNS}

Jusque dans les années 1960, les populations rurales de l'Arapiuns (dernier affluent du Tapajós, sur la rive droite du bas Amazone) occupaient l'espace sous la forme de groupes résidentiels dispersés en hameaux, au bord des lacs formés par les méandres de la rivière ou le long des cours d'eau à l'intérieur des terres. Sur l'initiative de commerçants ou de patrons locaux ${ }^{4}$, de petits villages groupés commencèrent à s'établir, dans les années 1960, autour de leur échoppe (freguesia). Ainsi, les premiers villages, qui prendront par la suite le nom de 
« communautés », furent souvent créés à l'initiative d'une famille liée à la ville et aux secteurs ecclésiastiques, dont était issue une figure charismatique - par exemple, un commerçant. Une parcelle de terre était alors stratégiquement mise à disposition de la collectivité par le groupe résidentiel auquel appartenait ce personnage-clé.

\section{Le terrain : balises topographiques}

L'Arapiuns est surnommé la « rivière noire » en raison de ses tonalités plus sombres que celles de l'Amazone. Ses eaux, en fait limpides, se déversent quasiment à la confluence du Tapajós et de l'Amazone. C'est une rivière de la faim, pauvre en phytoplancton, où la pêche ne s'est pas développée comme une activité lucrative. Hors période de piracema ${ }^{5}$, celle-ci fournit à peine les protéines nécessaires à l'alimentation des nombreux riverains. Pour la plupart, ce sont avant tout de petits agriculteurs, spécialisés dans la production de farine de manioc pour l'autoconsommation. Des activités extractives (chasse, cueillette) permettent de compléter le menu quotidien ou peuvent être réalisées à des fins lucratives : c'est le cas de la récolte du latex ainsi que de la confection d'artisanat en palmes. Ces activités se répartissent tout au long de l'année, selon le calendrier du cycle aquatique.

De janvier à juillet, les eaux montent jusqu'à immerger une grande partie de l'espace terrestre. Lors des années de grandes crues, elles viennent frôler dangereusement le seuil des maisons construites à même le sol, sur la terre ferme. Lorsque des habitations sont éloignées du village, par exemple lorsqu'elles sont construites près des abattis, elles se retrouvent alors isolées, n'étant plus accessibles qu'en barque. D'août à décembre, l'étiage laisse apparaître de grandes plages de sable blanc et une petite végétation arbustive, avec des « lacs » qui se forment entre les méandres. C'est alors le temps de l'abondance, des visites entre amis et des piracaias sur la plage, à la nuit tombée, moments privilégiés où l'on cuit sur un foyer improvisé le poisson pêché collectivement pendant la journée.

La plupart des habitations sont construites en palmes ou en bois, parfois une combinaison des deux, selon les ressources du foyer. Plus récemment, les briques et tuiles d'argile ont été introduites mais seule une minorité de maisons en sont pourvues. La majorité des villages de l'Arapiuns ne possède pas encore l'électricité, mais cela ne saurait tarder. Le Gouvernement a mis en place un ambitieux projet, Luz para todos, dont l'exécution a déjà commencé dans la région ${ }^{6}$. L'accès à la ville la plus proche, Santarém, s'effectue uniquement par voie fluviale. Le bateau passe deux fois par semaine et réalise le trajet en quelques heures depuis les villages de l'aval. Sur les deux rives, des dizaines de petites chapelles apparaissent, indiquant la présence des « communautés ». 


\section{Des petits hameaux dispersés entre les méandres de la rivière...}

Une des caractéristiques du peuplement de l'Arapiuns est sa densité relativement faible. Ici, pas de villes riveraines, ni même de vilas (« agglomération-siège du district, celui-ci étant la plus petite unité politique territoriale, sous-division du município » [Araújo 2009, §3]), juste quelques petits villages ${ }^{7}$ formés de groupes résidentiels. À la fin $\mathrm{du} \mathrm{XIx}^{\mathrm{e}}$ siècle, les riverains entretenaient déjà des liens économiques réguliers avec la ville de Santarém, où ils écoulaient leur production (farine de manioc, corde de curauá [Ananas erectifolius], palmes pour la construction, latex, vannerie, etc.). Les voyages, plus ou moins fréquents, duraient plusieurs jours et étaient réalisés en famille, au moyen d'une barge à voile (batelão). Le long de la rivière, les groupes résidentiels, fondés sur des relations de parenté et autour d'unités de production, occupaient et aménageaient de vastes territoires. Ils vivaient, au quotidien, dans un isolement relatif. Les festivités religieuses étaient l'occasion bienvenue de ripailler avec les amis, compères et connaissances d'autres groupes résidentiels plus éloignés. Ces fêtes étaient organisées par le «propriétaire » de l'image pieuse ${ }^{8}$ et entièrement à sa charge. Elles étaient l'occasion, pour les hôtes, d'accumuler du «capital symbolique » (Bourdieu 1980), en offrant boisson, repas et divertissement aux convives, et favorisaient la rencontre de jeunes gens de groupes résidentiels distincts pour éventuellement y sceller des promesses d'union. Ainsi, jusque dans les années 1960, les villages (appelés aujourd'hui « communautés ») n'existaient pas dans l'Arapiuns. On y trouvait seulement des «lieux» dominés par des « familles », c'est-à-dire les territoires des groupes résidentiels. Le commerce de biens de consommation en provenance de la ville était principalement assuré par des commerçants navigants, les regatões.

\section{... aux premières communautés}

À quelques heures de marche de là, dans la várzea, au bord du Lago Grande et à Arapixuna (Figure 1), l'occupation pérenne de certains lieux par des parentèles déterminées est plus ancienne ${ }^{9}$ et la situation un peu différente. Les commerçants étaient implantés depuis longtemps, entourés de leur freguesia. En effet, depuis l'époque coloniale et l'installation par les jésuites de la mission des Indiens tapajó à l'emplacement de la ville de Santarém (1616), les colons portugais avaient élu la várzea, très fertile, comme lieu privilégié pour l'installation de leurs exploitations (plantations de cacao, élevage bovin, commerce des épices et du poisson séché). La prospérité des colons était bâtie sur l'utilisation intensive de la main-d'œuvre indigène semi-servile présente dans les missions, lesquelles allaient devenir des vilas suite à la proclamation du Directoire ${ }^{10}$ et à l'expulsion des jésuites du Brésil (1755). La région de várzea de Santarém - alors 
appelée « couloir du cacao »- jouait un rôle stratégique dans le développement des exportations de la Colonie. Pour encourager la production, la Couronne portugaise y avait distribué de nombreuses sesmarias ${ }^{11}$. Après l'abolition du Directoire (1798), une partie des populations les plus modestes, principalement les Indiens (désormais émancipés) et les métis, caractérisée par une forte mobilité (Harris 2010), se dispersa le long du Tapajós et de l'Arapiuns (la várzea étant déjà occupée par les propriétaires terriens), formant de petits hameaux. D’autres s'établirent dans la várzea au service de planteurs et d'éleveurs. Enfin, une partie s'enrôla dans les corps de milice de l'armée.

C'est dans ce contexte qu'une partie de ces populations s'engagea dans les conflits armés de la Cabanagem (1832-1840), une révolte régionale surgie peu après la proclamation de l'indépendance du Brésil. Les localités situées en aval de l'Arapiuns furent le théâtre de violents conflits. Cuipiranga (Figure 1) fut ainsi l'un des bastions emblématiques des révoltés, et Vila Franca, une base de repli des forces légales (Arquivo público do Estado do Pará 1837; Barriga 2007 ; Harris 2010). Les récits de fuite de familles entières, parties se cacher à l'approche des insurgés (cabanos), décrits comme cruels et sanguinaires, sont encore aujourd'hui largement répandus, faisant figure de mémoire collective. Une partie de ces mouvements migratoires serait à l'origine des noyaux de peuplement dispersés à l'intérieur des terres et aurait façonné les prémisses de l'occupation de l'interfluve Arapiuns-Lago Grande (O’Dwyer 2005; Ioris 2009). La période suivante constitua un moment-clé dans la "caboclisation" (Nugent 1993) des populations rurales amazoniennes, notamment avec la fixation de la langue portugaise aux dépens du nheengatu (Borges 1994). Dès 1850, la région fut mobilisée par le boom du caoutchouc. L'entrée dans le $\mathrm{xx}^{\mathrm{e}}$ siècle de la population locale se caractérisa par sa participation aux différents cycles économiques ainsi que par une forte mobilité.

\section{Vila Brasil, une " communauté " riveraine}

Faisons escale à Vila Brasil et à Arimum, sur la rive gauche du bas Arapiuns. $\mathrm{Au}$ début $\mathrm{du} \mathrm{xx}^{\mathrm{e}}$ siècle, une dizaine de groupes résidentiels s'étaient réparti l'occupation du territoire correspondant aujourd'hui à ces deux «communautés ». Celui-ci s'étend du lac Camuci en aval au cap d'Aratapi en amont (soit sur $31 \mathrm{~km}$ en suivant le cours de la rivière). Il est bordé à l'est par l'Arapiuns et se prolonge à l'ouest à l'intérieur des terres jusqu'à l'actuelle route Translago, dans la zone interfluviale, en direction du Lago Grande (Figure 2). Les habitants de ces villages sont unis par des liens de parenté et une histoire commune, constituant un " système social homogène » (Menget 1985). Néanmoins, sous une apparente homogénéité, certains groupes résidentiels se sont distingués des autres dès les débuts par un accès privilégié à des formes de pouvoir institutionnalisées (comme l'obtention de documents fonciers ${ }^{12}$ ) et un capital symbolique plus important. 
Ceci se traduisit, par exemple, par le statut supérieur qui fut attribué aux groupes résidentiels détenteurs de bétail même si, objectivement, leurs conditions de vie ne différaient guère de celles de leurs voisins. L'occupation du territoire était donc soumise, dès l'origine, à des représentations locales où coexistaient des différentiations de statut et de classe. Le positionnement et les stratégies de reproduction mis en œuvre au sein des groupes pouvaient varier selon les schèmes de perception $\mathrm{du}$ pouvoir et du territoire propres à chacun.

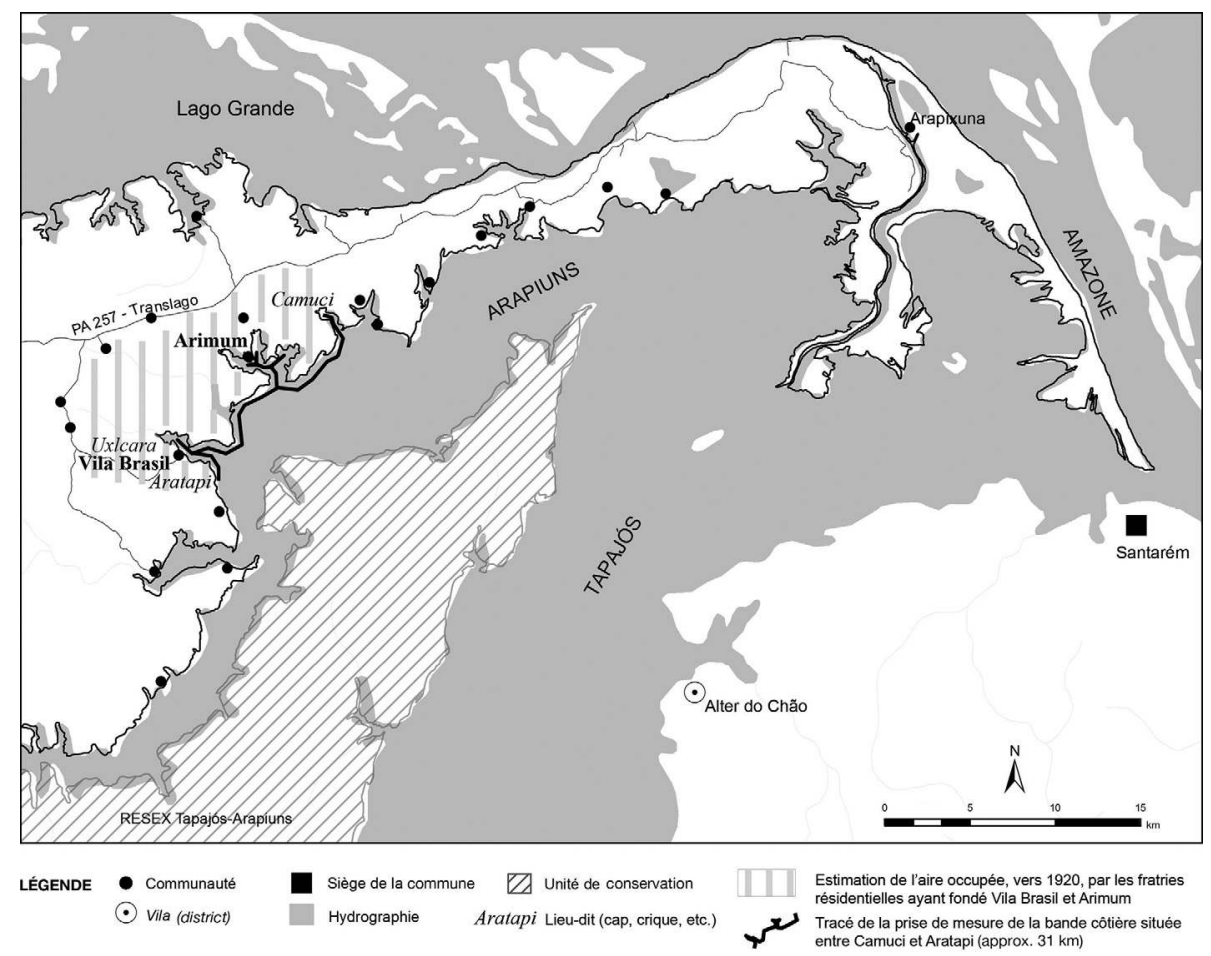

FIG. 2 - Répartition approximative des groupes résidentiels et communautés.

Les habitants actuels de Vila Brasil racontent qu'en 1952 dona Vitória ${ }^{13}$ et son groupe résidentiel occupaient le lieu-dit Uxícara, au fond d'une crique. Un beau matin, João Raymundo Delgado arriva de Belterra dans sa barge à voile. Habile orateur et commerçant dans l'âme, il se lia d'amitié avec les familles du voisinage et décida de s'y établir. Il vécut dans un premier temps chez un groupe résidentiel proche, au cap Aratapi (Figure 2). Puis il épousa Francisca, petite-fille de la doyenne du groupe résidentiel d'Uxícara. Cette union lui permit de s'intégrer rapidement dans le tissu social local. Cinq ans après son arrivée, il fit planter un champ de maïs sur le territoire de ses beaux-parents à l'emplacement d'un 
tucumãzal $^{14}$, actuel terrain de football de Vila Brasil. Plus tard, il fit construire un barracão, salle communale destinée à accueillir les activités religieuses, l'école et les fêtes auxquelles étaient conviés l'ensemble des groupes résidentiels des environs. En 1959, João Raymundo participa à la première semaine de catéchèse annuelle de Santarém, destinée à former les catéchistes de la zone rurale. À partir de cette date, le « lieu » Uxícara fut reconnu officiellement comme " communauté » par l'Église et les groupes résidentiels locaux. Au début des années 1960, une école radiophonique du Mouvement ecclésial de base (MEB) fut installée dans le village pour l'alphabétisation des adultes. Une église fut construite et João Raymundo ouvrit un petit commerce de produits manufacturés. Il était devenu, entre-temps, le premier président de la communauté, laquelle avait pris officiellement le nom de Vila Brasil.

Ce cas est emblématique en ce qu'il est l'exemple type de la formation communautaire autour de la figure du commerçant. C'est l'« homme fort rural » ( "rural "big man” ") décrit par Gross (1973, p. 123), « qui combine l'argent qu'il a gagné avec l'agriculture et/ou le commerce avec des contacts stratégiques au sein des autorités locales, étatiques ou fédérales dans le but de se bâtir une base locale de pouvoir ». Le processus habituel, selon l'auteur, consiste à ouvrir une échoppe rurale autour de laquelle vient se greffer un village. À travers des relations paternalistes et clientélistes qu'il entretient avec ses clients et/ou employés, le commerçant maintient un contrôle social sur eux. Il commence à discuter de la nécessité d'avoir une chapelle afin de créer du lien social dans la localité, sans même évoquer les clients potentiels que cela attire les jours de célébration. Déjà dans les années 1940, Galvão (1976 [1955], p. 9) décrivait la communauté de Gurupá (bas Amazone) comme une " unité territoriale socio-économique et religieuse » composée d'une freguesia, de sítios et de vizinhanças (respectivement « clientèle », « lieux de production» et "voisinages »). Il s'agissait donc de petits agglomérats de population extractiviste, rarement composés de plus de 150 individus (soit environ 25 familles), où se trouvait l'entrepôt d'un commerçant. La vie des riverains s'articulait autour de deux institutions-clés : la figure du commerçant et son échoppe, et les confréries religieuses organisées autour du culte d'un saint.

\section{LE RÔLE DE L'ÉGLISE CATHOLIQUE DANS LA GENÈSE DES « COMMUNAUTÉS »}

Au début des années 1970, Vila Brasil comptait 380 habitants (Prelazia de Santarém 1970-1975), soit une soixantaine de familles. Le commerçant était devenu le principal acheteur de latex de la région et il est remémoré comme un homme populaire, jovial et généreux. Lorsque les visites des prêtres devinrent plus fréquentes, il encouragea les groupes résidentiels éloignés du village à construire une église à Arimum, localité voisine. Jusqu'à cette date, ces groupes se 
déplaçaient jusqu'à Vila Brasil, au prix de plusieurs heures d'efforts, pour participer aux cérémonies religieuses et à d'autres activités. L'église fut construite en 1973 et une taverne fut ouverte et confiée à l'un des jeunes apprentis du commerçant. C'est ainsi qu'une quinzaine de familles « quittèrent » Vila Brasil pour former la communauté d'Arimum.

La décennie de 1970 correspond en effet dans la région de Santarém à la mise en ouvre par l'Église catholique de la théologie de la libération, une idéologie destinée à permettre l'émancipation (spirituelle, économique et politique) des populations rurales pauvres, trop souvent en proie à des rapports de force défavorables. Relayés localement par des hommes charismatiques, les prêtres encouragèrent dans toute la région - y compris la várzea (O’Dwyer 2005) - la formation de communautés ecclésiales de base (CEB), organisées sous la forme d'un village construit autour d'une chapelle. C'est ainsi que, dès les « années 1960, l'Église catholique s'approprie le terme [communauté], dans un moment de réorganisation de ses structures, pour incarner une présence missionnaire plus effective dans différentes réalités locales qui coexistaient à l'intérieur d'immenses territoires paroissiaux, en lui donnant des significations nouvelles par le biais d'une association entre communauté et ecclesia » (Araújo 2009, § 2).

\section{La mise en place des communautés ecclésiales de base (CEB) et la formation des vilas}

La prélature de Santarém avait été créée dès 1903 mais, faute de prêtres, ce n'est qu'à partir des années 1960 que les visites paroissiales reprirent dans la région de l'Arapiuns et du Lago Grande ${ }^{15}$. Les prêtres, lorsqu'ils visitaient les hameaux, officiaient dans des chapelles privées dressées pour l'occasion par l'un ou l'autre des groupes résidentiels souhaitant faire célébrer des baptêmes. Cette époque, marquée par l'essor de la théologie de la libération et l'organisation des CEB, correspond à la création des premières vilas. Il s'agissait d'un espace collectif destiné à accueillir, en habitat groupé, les familles souhaitant fonder une communauté spirituelle et morale. La « communauté » était donc avant tout un concept idéel dont la seule manifestation concrète était la construction d'une vila entendue comme un agglomérat villageois. Une petite chapelle y était érigée et les familles de l'ensemble des groupes résidentiels des environs pouvaient y construire une maison et participer aux activités religieuses.

Avec la théologie de la libération et sa « préférence pour les pauvres », l'Église adopta un répertoire mobilisateur de religiosité politisée, où les thèmes de la lutte pour la terre et la valorisation de la vie communautaire étaient déclinés à partir de codes moraux et d'une allégorie de la sortie d'Égypte en tant que fin de la servitude (Houtzager 2004). Ce discours était véhiculé par les Semaines de catéchèse annuelles (initiées à Santarém en 1959), auxquelles étaient invités les dirigeants et futurs dirigeants communautaires. Les efforts de conscientisation de l'Église ${ }^{16}$, conjugués à partir des années 1980 à ceux du Syndicat des travailleurs 
ruraux (Leroy 1991) ${ }^{17}$, martelèrent inlassablement les idées d'émancipation du travailleur rural (« Lève-toi et marche », Actes $3: 6$ ).

Ainsi, la communauté a été, dès son origine, conçue comme un élément fédérateur d'individus, rassemblés autour de principes moraux et religieux, en vertu desquels chacun espère entraide et solidarité. Le modus operandi institué par les instigateurs des CEB pour l'organisation des travaux communautaires d'entretien de la vila en est un exemple : il s'agissait de transférer dans la sphère communautaire le système du mutirão ou puxirum (travaux agricoles réalisés collectivement par les unités de production). Or, ce qui était pensé comme collectif dans un cas (réaliser tous ensemble l'entretien régulier des espaces communautaires) était en fait considéré comme un service rétribué dans l'autre (les journées de travail dans les abattis sont échangées réciproquement entre les groupes domestiques).

\section{La réorganisation communautaire de l'espace}

Grâce à l'appui de relais locaux (commerçants, etc.), l'Église catholique contribua, en la justifiant moralement, à la concentration des populations riveraines jusqu'ici éparses, en les regroupant autour d'une CEB et d'un ensemble d'infrastructures réputées collectives (salle communale, église, école). La formation de villages en habitat groupé répondant à une idéologie « moderne » modifia sensiblement l'approche du territoire et la conception de l'espace, puisque les familles issues de groupes résidentiels distincts (et jusque-là autonomes) étaient désormais destinées à vivre côte à côte sur un lopin de terre réputé communautaire. L'urbanisme y reproduisit une segmentation spatiale en groupes résidentiels de telle sorte que les « quartiers » du village correspondaient aux unités domestiques possédant leurs abattis dans un même lieu. D'ailleurs, ces groupes n'en continuèrent pas moins à occuper leur territoire d'origine (les « lieux » transmis de génération en génération), où se trouvaient leurs plantations. Aujourd'hui encore, les familles passent la semaine dans le tapirizinho ${ }^{18}$, localisé près des jardins, et le week-end dans la "maison », au village. Une hiérarchisation s'est ainsi opérée entre la vila, village d'habitat groupé nouvellement créé, et les « centres », c'est-à-dire les territoires des groupes résidentiels où se trouvent toujours leurs espaces de production. Tout oppose ces espaces, à commencer par l'existence (ou non) d'une chapelle. Des couples antinomiques caractérisent la vila et le centre, tels que centralisé/dispersé, collectif/individuel, lieu de sociabilité/lieu de travail, éducation/ignorance, espace propre/boisé ${ }^{19}$, jusqu'à des conceptions essentialistes telles que moderne/arriéré, voire même civilisé/sauvage. Faulhaber propose l'idée d'un « centre » opposé au « bord » (lac, fleuve, route). Le premier « renvoie à des représentations liées à des situations de danger entraînées par le contact avec la nature incontrôlée » (1986, p. 397). La moralisation des concepts associés à l'idée de communauté n'est pas étrangère 
à son origine religieuse et prosélyte. D'ailleurs, l'initiative de créer une communauté est souvent attribuée à un individu sabio (savant) extérieur s'étant installé de façon durable dans le paysage local (entrosado). Ainsi, la formation du village suggère, dès ses débuts, une reclassification sociale et une évaluation morale de l'espace et de ses occupants.

\section{La formation des communautés par scission}

Aujourd'hui, on considère que l'espace géographique correspondant à la « communauté » comprend l'ensemble des terres des groupes résidentiels (les « centres ») qui lui sont affiliés administrativement. En raison de l'arrivée de nouveaux adhérents et de la sortie potentielle d'autres, notamment lorsque surgissent des disputes internes, les frontières de la " communauté » sont donc mobiles au gré des évènements régissant les relations entre les groupes qui la constituent. Ceci s'explique par l'idée que s'en font les riverains, pour qui elle représente une affiliation politique pragmatique (qui permet de scolariser ses enfants, de s'inscrire pour recevoir les allocations sociales, etc.) davantage qu'un système organisationnel discret possédant une logique fonctionnelle qui lui est propre. En effet, lors de sa création dans les années 1960, la «communauté » est venue se superposer à une répartition territoriale préexistante (celle des groupes résidentiels) et n'a jamais fonctionné comme une circonscription préétablie (à l'image des cantons paroissiaux), mais en fonction de l'adhésion des familles à un groupe spirituel, c'est-à-dire de leur participation aux activités religieuses (mais également sportives, festives, etc.) et au suivi de règles de conduite édictées par les dirigeants du village, à savoir, le " président de la communauté » et les catéchistes (pouvant être issus d'autres groupes résidentiels). En cas de désaccord, il était ainsi possible de cesser de participer à une « communauté » et de se tourner vers celle d'un beau-frère ou d'un compère située dans le voisinage.

Changer de " communauté » ou en créer une nouvelle n'impliquait pas de migration à proprement parler. C'est seulement le centre décisionnel des dissidents qui était délocalisé. Il s'agissait donc d'un groupe pratique qui se constituait autour d'un lieu de culte. Entre 1960 et 1970, une vingtaine de communautés furent créées le long de l'Arapiuns (Prelazia de Santarém 1970-1983) et les commerçants jouèrent un rôle actif dans ce processus. Chaque nouveau village rendait possible l'installation d'une taverne, sorte d'épicerie-comptoir où seraient vendus ou échangés des biens de consommation importés de Santarém. Les prêtres et les commerçants encourageaient les groupes résidentiels des " centres » éloignés à fonder leur propre "communauté » et lieu de culte. Ces micro-villages étaient alors initialement formés par un groupe de parents, auquel viendraient s'agréger, avec le temps, d'autres familles.

Dans les années 1990, une nouvelle vague de «communautés » vit le jour, principalement issues de groupes résidentiels qui ne se sentaient pas représentés 
dans celles créées dans les années 1970. Suivant le principe du «marcher de ses propres pieds", ils s'autonomisèrent en se repliant sur leur territoire (le " centre ») pour fonder une nouvelle unité politico-religieuse. C'est donc à partir d'alliances entre groupes résidentiels et de scissions que se formèrent, en deux temps, dans les années 1970 puis 1990, les villages de l'Arapiuns (Figure 3). Les toponymes locaux conservent encore la mémoire de ces dynamiques, comme dans les cas de Sociedade dos Parentes et Nova Sociedade dos Parentes; Bacuri et Bacurizinho ; Tucumã et Nova Sociedade do Tucumã ; São José I, II et III ; Mentai, Santa Rosa do Mentai et Alto Mentai ; São Francisco (Gurupá) et Novo Gurupá. Au bord du Lago Grande, on trouve, selon le même principe : Peré Salvação, Peré Boa Vista, Paraíso do Peré et São José centro do Peré ; ou encore Jacaré, Jacarezinho, Jacarezão, etc. Aujourd'hui encore, les disputes de pouvoir et de prestige qui ne trouvent pas d'issue peuvent se solder par la création d'un nouveau village.

\section{L'ARRIVÉE DES INSTITUTIONS DE L'ÉTAT ET L'ENFERMEMENT CONJONCTUREL}

Depuis la période coloniale, l'occupation territoriale de l'Amazonie brésilienne s'est principalement réalisée à travers une occupation usufruitière (posse) ${ }^{20}$ de terres publiques, considérées comme « libres », car non occupées. Cette pratique se situait en marge du système juridique et l'occupation foncière se réalisait par appropriation de terres publiques par des posseiros, sans délivrance de titres de propriété (Silva 2008). Les documents de déclaration d'occupation (les declarações de posse) faisaient d'ailleurs figure d'exception. La relation juridique que ces posseiros maintenaient avec leur terre changea radicalement à partir de $1964^{21}$, lorsqu'un nouveau modèle de développement pour l'Amazonie fut instauré. Son objectif était la mise en valeur économique de la région. Il prévoyait la réalisation d'un ample diagnostic foncier afin de cibler stratégiquement la destination des terres publiques (projets de réforme agraire, exploitation économique, colonisation).

En 1970, 1'Institut national de réforme agraire (Incra) fut créé avec pour mission la régularisation foncière des posses par l'émission de titres de propriété et la collecte d'un impôt sur la terre. Un bureau de l'Incra fut installé à Santarém. En 1977, cet organisme s'attela à un programme d'enregistrement au cadastre de l'ensemble des terres comprises dans l'interfluve Arapiuns-Lago Grande, incluant la région d'Arapixuna (Figure 1). Ce vaste ensemble territorial prit alors la désignation de gleba Lago Grande da Franca ${ }^{22}$. Des techniciens réalisèrent des relevés topographiques sur place afin de recenser les aires occupées.

Dans le bas Arapiuns, face à l'arrivée d'acteurs extérieurs attirés par le marché des terres dans la région et sur les conseils des agents de l'Église et du Syndicat, de nombreux habitants se mobilisèrent pour déclarer l'occupation de leurs terres. 
La nouvelle politique foncière influença les mobilisations politiques et sociales pour le droit à la terre. Certains se remémorent cette période de grands bouleversements en évoquant leurs actes de résistance pour défendre leurs terres menacées d'invasion par des exploitants agricoles enrichis lors des cycles économiques antérieurs ; d'autres se souviennent plutôt des nouvelles expériences agricoles qui furent alors menées (plantations de cupuaçu [Theobroma grandiflorum], tabac, café). Mais pour tous, elle a signifié, au cœur des groupes résidentiels, l'adoption de stratégies visant à mettre en adéquation les limites des territoires traditionnels (les « centres ») avec les nouvelles exigences normatives du droit foncier imposant la délimitation de lots individuels destinés à être privatisés.

Jusqu'à aujourd'hui, l'arrivée de l'Incra dans la région marque une rupture entre un «avant» et un « après » qui est invariablement énoncé par la formule suivante : « Aujourd'hui, tout a un propriétaire. » Cette formule met l'accent sur deux aspects : premièrement, l'existence de tensions au cœur des groupes résidentiels ; deuxièmement, la fin des «terres libres ». Dans les deux cas, elle laisse transparaître une sensation d'enfermement due aux nouvelles contraintes foncières. Avant le passage de l'Incra, il était possible, nous a-t-on dit, de planter un jardin ou de construire une maison là où cela semblait le plus adapté, sans avoir de comptes à rendre. Depuis l'arrivée de l'Incra, chaque parcelle est nommée et cadastrée comme posse, et les usufruitiers (posseiros) sont contraints de s'installer dans les limites du lot enregistré.

Cette représentation est à comprendre dans le cadre des dynamiques d'occupation des groupes résidentiels. En principe, avant 1970, dans les «centres », les voisins étaient tous parents puisqu'ils étaient membres du même groupe résidentiel. Les couples pouvaient donc, dans les limites du territoire familial, s'installer où ils le souhaitaient, la néo-localité étant de rigueur. Ce que nos interlocuteurs déplorent aujourd'hui, sans le dire ouvertement, n'est pas l'occupation individuelle de l'espace (qui existait déjà puisque les espaces d'habitation et de production étaient " privatifs »), mais plutôt l'idée de privatiser d'avance une fraction du territoire du groupe résidentiel, puisque le lot déclaré inclut forcément des espaces encore vierges (notamment de forêt), dont l'utilisation future est projetée.

Cette configuration impose ainsi une rigidification structurelle de l'occupation de l'espace au cœur des groupes résidentiels : d'avance, tout l'espace du groupe se trouve quadrillé et cette fragmentation est accompagnée d'une attribution des parcelles aux déclarants qui en deviennent - dans les représentations locales - les « propriétaires » (donos), quand bien même juridiquement ils n'en sont que les usufruitiers (posseiros). Dès lors, pour une famille déjà constituée, devoir quitter sa terre (jusqu'à aujourd'hui, les conflits au sein des familles sont résolus par des migratoires temporaires ou définitives) représente désormais un drame familial puisqu' « il n'y a plus de terres libres » et que, sans terre, il faut travailler pour d'autres. Cette situation de familles sans terre ${ }^{23}$ est sans doute 
l'une des conséquences les plus directes des stratégies d'appropriation foncière mises en œuvre après le passage de l'Incra. En 2012, cette situation de grande dépendance sociale concernait $22 \%$ des familles (Figure 4). À cet égard, la vila joua et joue toujours un rôle essentiel d'accueil et de mise à disposition d'infrastructures basiques.

À l'époque des travaux de l'Incra, la nouvelle situation juridique n'empêcha en fait pas les anciennes pratiques informelles d'occupation et de partage du foncier au sein des groupes résidentiels de se poursuivre. Le chef de famille choisissait parmi ses enfants ceux à qui il transmettrait le droit de s'établir sur le « centre » du groupe résidentiel. Les élus recevaient une parcelle et il était attendu des autres qu'ils intègrent un autre groupe résidentiel. Lorsqu'ils n'y parvenaient pas, ils devenaient des agrégats plus ou moins tolérés, sans jamais accéder au statut de membre légitime du groupe. La législation agraire brésilienne exige cependant que chaque agriculteur en activité puisse bénéficier du droit d'usufruit sur le lopin de terre qu'il cultive s'il s'agit d'une terre publique (c'est-à-dire ne possédant pas de propriétaire légal). Or, dans l'Arapiuns, seuls les membres légitimes des groupes résidentiels étaient localement considérés comme « maîtres » (donos) des lieux, pas leurs agrégats. La logique législative interfère donc directement dans un domaine traditionnellement réservé au chef de famille et cette problématique a resurgi dans les années 1990 et 2000, lorsque la première génération à avoir enregistré l'usufruit de ses terres à l'Incra a transmis son patrimoine territorial à la suivante.

Nous avons par exemple eu connaissance d'épisodes où les parents avaient enregistré la terre uniquement au nom de leurs filles mariées, à qui ils la destinaient. Le fils qui vivait sur le lopin des parents avec son épouse s'en aperçut et c'est le fonctionnaire de l'Incra qui dut arbitrer la querelle, attribuant de plein droit un lot au ménage du fils, contre l'avis du père. Les disputes d'héritage au sein des familles changèrent donc de forme et d'intensité.

Les mesures de l'Incra eurent aussi des effets visibles sur l'environnement et l'usage du sol. Dans la gleba Lago Grande da Franca, le recensement par l'Incra des terrains occupés avait été réalisé principalement en deux temps. Dans les années 1970, des topographes arpentèrent l'interfluve pour repérer les occupants usufruitiers (posseiros) ainsi que d'éventuels propriétaires. Dans les années 1980 et 1990, ils retournèrent dans les villages pour proposer aux premiers la régularisation foncière de leurs terres, distribuant à cette fin des licences d'occupation ${ }^{24}$. À cette occasion, les agents publics encouragèrent les habitants à rendre visible la mise en valeur de leur terrain enregistré en déboisant de grandes étendues de forêt. La conception développementiste du régime militaire visait à en finir avec une forêt jugée rétrograde (Araújo et Léna 2010). En 1984, les familles de Vila Brasil reçurent leurs licences. Beaucoup suivirent les recommandations et défrichèrent, plantèrent, firent des pâtures, mettant le feu à la forêt, espérant ainsi recevoir des documents de propriété. Une de ces familles aurait 
déboisé, à elle seule, près de 30 ha en un été, alors qu'elle n'en aurait normalement ouvert que deux sur la même période. Cette situation, consolidée à la fin des années 1980, présageait déjà les conflits à venir, puisque la compétition pour l'accès à la terre et les pressions socio-environnementales s'étaient accrues.

\section{L'ORGANISATION SOCIALE EN FRATRIES RÉSIDENTIELLES : MODALITÉS D'OCCUPATION TERRITORIALE ET PRATIQUES DE TRANSMISSION DU FONCIER}

Nous avons vu que les « communautés » se sont constituées à partir de la participation de groupes résidentiels solidaires qui occupaient préalablement de vastes territoires (devenus des «centres» de villages). Ceux-ci, organisés en fratries résidentielles, se perpétuent au moyen de stratégies fondées sur l'exclusion. Des pratiques variées de transmission du foncier sont dès lors mises en œuvre, dont l'enjeu principal consiste à «désapparenter » une partie des descendants pour accueillir les conjoints des frères et sœurs restants, permettant ainsi la reproduction du groupe sur un territoire circonscrit.

\section{Les fratries résidentielles : un groupe familial solidaire}

Dans l'Arapiuns, l'organisation sociale et territoriale des groupes résidentiels se fonde, aujourd'hui encore, sur ce que nous appellerons des fratries résidentielles. Celles-ci se définissent par deux éléments associés : une composante territoriale et une « famille ». Ainsi, un territoire aux contours relativement stables dans le temps, initialement fixés par un fondateur, est dominé par plusieurs unités domestiques, toutes apparentées au fondateur et par là également liées entre elles. Chaque unité domestique, composée d'un couple et de ses enfants célibataires, occupe une maison et possède ses propres abattis. Les unités domestiques sont solidaires et coopèrent entre elles en s'échangeant des denrées et, lors des puxirum, des jours de travail. La « fratrie résidentielle » désigne donc un groupe de parents uni autour d'un patrimoine commun (le territoire, le nom et le capital symbolique transmis par le fondateur) mais non collectif : chaque unité domestique possède sa propre maison, ses propres abattis et une certaine autonomie dans sa gestion. Cette autonomie économique et résidentielle est relative, puisqu'elle opère à l'intérieur d'un réseau et d'un territoire intra-familiaux et est soumise, à ce titre, aux intérêts de la fratrie. La filiation est bilatérale et les descendants du fondateur, incluant plusieurs générations de collatéraux, revendiquent leur appartenance au groupe et à son territoire. A priori, tous briguent le statut d'héritiers. Nous verrons plus loin quels arguments sont alors avancés.

À chaque nouvelle génération, les frères et sœurs sont mis en concurrence pour la transmission d'un lopin de terre par leurs parents. Lorsqu'un ménage se forme, il va vivre auprès des parents de l'homme ou de la femme jusqu'à recevoir 
(ou non), en général au bout d'environ cinq ans et après la naissance de plusieurs enfants, l'autorisation de choisir - selon le principe de néo-localité et dans les limites du territoire du groupe résidentiel - une nouvelle parcelle de terrain où construire sa maison et planter un jardin. Les jeunes couples ne vivent cependant pas dans la même maison que les parents. Tant qu'ils n'ont pas reçu de terrain, ils restent dépendants d'eux pour l'accès aux abattis, aidant à cultiver ceux des parents. Ils partagent dès lors aussi la même cuisine, ainsi du reste que toutes les formes de revenus, mais ils ont d'emblée une maison distincte.

Lorsque l'on arrive dans les actuelles communautés, l'organisation sociale en fratries résidentielles saute aux yeux, puisque les frères et sœurs d'une même « famille » (au sens défini plus loin) vivent les uns à côté des autres, avec leurs descendants, recréant ainsi des sortes de quartiers villageois qui correspondent à la répartition des maisonnées dans les «centres ». En effet, la relation sociale pertinente pour appréhender les enjeux de ce système est la germanité. Au départ, des frères et sœurs et leurs conjoints occupent un territoire défini. Il s'agit alors d'une fratrie. De par son cycle de développement (Goody 1975), dès la génération suivante, le groupe inclura des cousins germains, et ainsi de suite, jusqu'à ce qu'il implose suite à des divergences, donnant naissance à plusieurs fratries politiquement autonomes. Dans ce cas, le nom du fondateur initial est oublié et la mémoire généalogique tronquée.

Seuls les membres légitimes de la fratrie résidentielle sont réputés faire partie de la « famille » (les exclus étant dès lors considérés comme des parents éloignés). Est membre de la «fratrie », le frère ou la sœur (par extension, le cousin ou la cousine) ayant vécu sur le territoire du groupe pendant l'enfance, puis qui y établit sa résidence une fois marié. C'est donc la résidence (et non la seule filiation) qui conditionne l'appartenance à la «famille » et l'accès aux droits fonciers. La transmission de la terre aux enfants qui restent vivre auprès de leurs parents garantit la continuité intergénérationnelle, puisque les petits-enfants prendront soin de leurs grands-parents. Ceci nous amène à proposer cette notion de « fratrie résidentielle ", où un groupe de germains habitant au même endroit maintient, sur le territoire de ses parents, une maîtrise reconnue et respectée par les autres habitants des environs. La notion de fratrie résidentielle recoupe donc deux aspects : l'appartenance de ses membres à une « famille » spécifique (incarnée dans la figure du fondateur) et leur occupation spatiale d'un espace déterminé. Elle implique une solidarité de principe entre les familles dans la défense d'intérêts communs; notamment territoriaux et politiques. Ceci est exprimé par l'emploi du suffixe « - zada » accolé au nom de référence - par exemple, la Balboazada, pour désigner la fratrie résidentielle Balboa (du nom du fondateur, même si ses descendants s'appellent désormais autrement) et les agrégats qui la soutiennent politiquement (familles sans terre dépendantes, notamment ; Figure 3).

La fratrie résidentielle se présente comme faisant bloc autour de pratiques et valeurs communes et exerce un contrôle sur un espace. Ce modèle renvoie à une 
Fratrie résidentielle $\mathrm{A}$

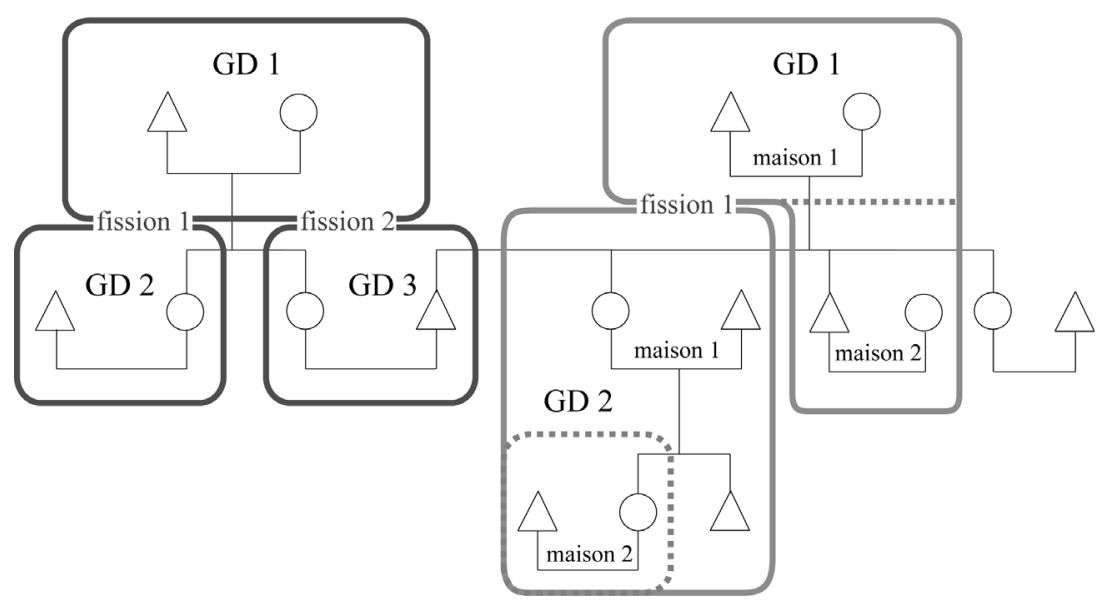

FIG. 3a-Cycle de développement des groupes domestiques (GD) et perpétuation des fratries résidentielles.

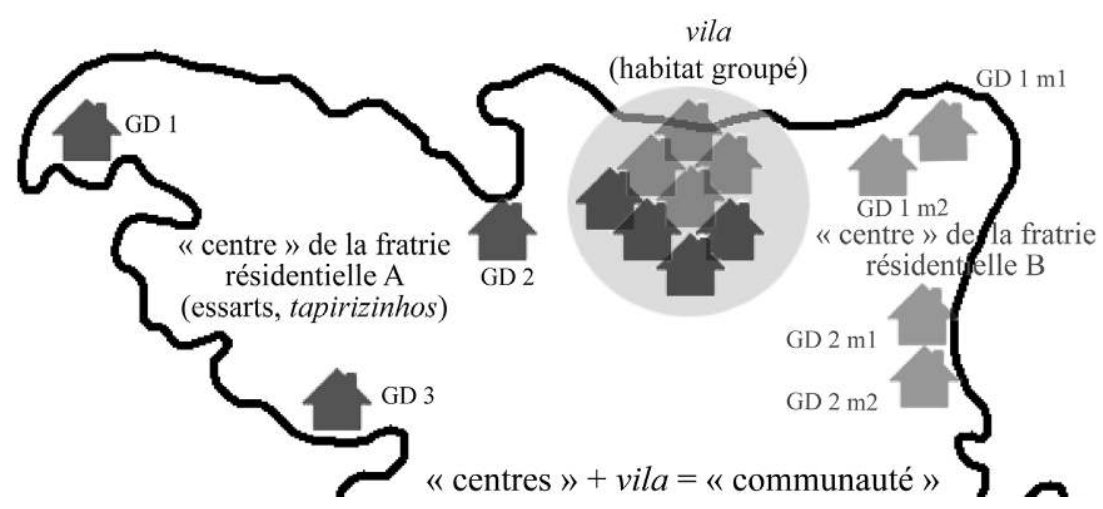

FIg. $3 b$ - Organisation spatiale des unités domestiques sur le territoire de la fratrie résidentielle et appartenance villageoise.

conception plus large du territoire, où celui-ci peut être associé à un réseau mobile et reconfigurable. En effet, les germains exclus ou qui se sont alliés à un autre groupe résidentiel dans une autre localité peuvent espérer nouer des relations politiques et économiques avec leurs "parents» restés sur la terre d'origine. Ces lieux s'égrènent tout le long de la rivière et balisent un espace parcouru par de 
possibles flux de personnes, d'artefacts et de biens symboliques. S'y inscrit notamment la pratique fréquente de migration temporaire ou définitive d'unités familiales, pour saisir une opportunité économique ou d'alliance, suite à une exclusion ou encore pour résoudre pacifiquement un conflit. Les migrations au sein de ce réseau alimentent le système des fratries résidentielles, qui peuvent le mettre stratégiquement à profit. C'est ainsi que le patriarche d'une famille nombreuse avait su habilement exploiter la pluri résidence rendue possible par les mariages réalisés par plusieurs de ses fils avec des femmes issues d'un même autre lieu (des sœurs ?) à $48 \mathrm{~km}$ en amont de l'Arapiuns. Lui-même détenteur de plantations d'hévéa en aval, il avait bâti une véritable entreprise commerciale par la réalisation conjointe d'une activité extractive en aval (la collecte du latex qu'il assurait avec ses enfants célibataires) et d'une activité agricole en amont (la culture à grande échelle de tabac, café, manioc et autres cultigènes, qu'il assurait en partenariat avec ses fils mariés). La vente de ces produits lui valut de figurer en $267^{\mathrm{e}}$ position parmi les 411 " propriétaires d'établissements ruraux » de Santarém lors du recensement économique de l’État du Pará en 1920 (República dos Estados federados do Brasil 1927) ${ }^{25}$.

\section{L'exclusion comme modèle de construction du socius}

Le frère ou la sœur qui part vivre définitivement sur les terres de la fratrie résidentielle de son conjoint est « désapparenté » et perd tout droit sur la terre de ses parents. Nos données suggèrent que les procédés qui visent à dissoudre les liens de parenté au sein des fratries sont indispensables à la reproduction sociale. Ceci vient nuancer d'autres études qui montrent que c'est la production de parenté qui construit le socius (Gow 1991). Reste encore à définir qui peut (ou non) s'établir sur le territoire familial.

La transmission du foncier se réalise entre un ascendant et certains de ses descendants mariés qui résident sur place. Les enfants célibataires n'ont, en effet, aucun droit : ils vivent chez leurs parents et cultivent le jardin de ceux-ci.

Des mécanismes sont mis en œuvre afin de favoriser la permanence de descendants sur le territoire familial tout en préservant son intégrité. L'idée de transmission accompagne celle d'alliance puisque les conjoints, en général issus d'autres fratries résidentielles, sont apparentés par le mariage. Chaque descendant marié a, en principe, droit à une parcelle. Or nous avons observé qu'environ 5 à 11 enfants par foyer atteignent l'âge adulte. Un contrôle raisonné de la démographie devient crucial pour assurer à chacun un accès aux ressources suffisant pour vivre. La concurrence directe entre germains rend fragile cet équilibre et elle explique que la fratrie, apparaissant comme solidaire vis-à-vis de l'extérieur, soit traversée par de nombreux conflits larvés, prenant souvent la forme d'accusations de sorcellerie entre proches parents. Tout l'enjeu consiste à ne pas faire partie des descendants qui seront « désapparentés ». 
Pour éviter l'effet de «trop-plein », des stratégies familiales sont mises en œuvre. Même si l'on peut dégager des tendances, toutes les fratries résidentielles ne procèdent pas de la même façon. Certaines, par exemple, ont adopté un système uxorilocal : les descendants de sexe masculin se marient à l'extérieur du groupe et les gendres sont accueillis. Cette pratique, caractéristique d'une société agricole - dont l'activité est plutôt féminine - semble avoir été largement adoptée le long de l'Arapiuns, au moins jusque dans les années 1970, comme en témoigne l'univers symbolique et discursif dans lequel se déroulent les transactions foncières et matrimoniales. L'attachement affectif qui lie les riverains à leur terre est corrélé, dans les discours, au fait que l'on enterre dans le jardin familial le cordon ombilical des nouveau-nés et le placenta de la mère. Les femmes sont vues comme des figures stables, garantes de la perpétuation du sentiment d'appartenance au lieu. À l'inverse, les hommes sont davantage décrits comme des acteurs qui circulent (entre les lieux, mais aussi entre les femmes). Il semble alors préférable d'accueillir des gendres et d'inciter les descendants de sexe masculin à se rapprocher d'une fratrie possédant des terres. D'après les récits, il était courant que la fille cadette « donne ${ }^{26}$ » à ses parents, dès la naissance, l'aînée de ses filles. Celle-ci s'occuperait d'eux sur leurs vieux jours et assurerait la permanence sur leur terre. L'héritier ultime est donc pensé comme une figure doublement féminine (la fille de la fille) et appartenant à la troisième génération. La transmission du sol repose ainsi sur une logique de descendance réputée « biologique », selon la rhétorique du sang. C'est ainsi que les anciens disaient toujours préférer les petits-enfants nés de leurs filles, héritiers indiscutables ${ }^{27}$ (et légitimes dans un système uxorilocal) à ceux nés de leurs belles-filles. Toutefois, ces représentations et pratiques ne correspondent pas à une prescription puisque, dans les faits, l'uxorilocalité n'était pas appliquée de manière rigide. Il semble davantage qu'elle s'accommode d'une certaine pragmatique dictée par l'état du cycle de développement du groupe au moment où est prise la décision de la fission de nouvelles unités domestiques (Goody 1975 ; Stoll 2014). De même, si de la terre était disponible chez les parents de l'homme, une belle-fille issue d'une famille sans terre rejoignait le groupe résidentiel de son époux. Dans ce cas, le couple ne jouissait pas d'une situation très enviable et devait fournir une contribution majorée à l'effort productif (travailler pour ses hôtes). Ces situations provoquaient des tensions, parfois des conflits entre belles-sœurs, pouvant mener au départ du couple. Par ailleurs, d'autres familles semblent davantage privilégier une approche virilocale, notamment celles d'éleveurs (une activité masculine) originaires de la várzea. Nos données ne nous permettent cependant pas de généraliser.

Dans l'ensemble, les pratiques résidentielles et de transmission du foncier ont subi des bouleversements récents après l'intervention de l'État en Amazonie. Au début des années 1970, alors même que l'Église opérait l'organisation en « communautés », l'irruption de l'Incra a remis en cause le modèle traditionnel d'occupation et de transmission de la terre. À la même période, on observe une 
importante croissance démographique, peut-être due aux progrès sanitaires et à la moralisation catholique de la vie conjugale (les prêtres incitent les couples à se marier à l'église et condamnent l'adultère, ce qui a pour effet une plus grande stabilité des noyaux familiaux et un accroissement du nombre d'enfants). Depuis lors, même si au sein des fratries résidentielles les principes qui ouvrent le droit à la transmission foncière demeurent les mêmes (faire partie de la « famille »), la disponibilité en terres libres est désormais le facteur déterminant dans le choix du lieu de résidence des ménages. À titre d'exemple, en 2012 à Arimum, sur 32 familles vivant de façon permanente sur leurs terres, $34 \%$ étaient uxorilocales (Figure 4).

\begin{tabular}{|c|c|c|c|c|c|}
\hline \multirow{2}{*}{$\begin{array}{c}\text { familles } \\
\text { uxorilocales }\end{array}$} & \multicolumn{3}{|c|}{ familles virilocales } & \multirow{2}{*}{$\begin{array}{c}\text { familles } \\
\text { sans terre }{ }^{b}\end{array}$} & \multirow{2}{*}{ intrus $^{c}$} \\
\hline & $\begin{array}{c}\text { maris } \\
\text { sans sœur }\end{array}$ & $\begin{array}{c}\text { épouses } \\
\text { sans terre }^{\text {a }}\end{array}$ & $\begin{array}{c}\text { accords } \\
\text { familiaux }\end{array}$ & & \\
\hline 11 & 3 & 8 & 3 & 4 & 3 \\
\hline $\begin{array}{c}\text { total }=11 \\
(34 \%)\end{array}$ & \multicolumn{3}{|c|}{$\begin{array}{c}\text { total }=14 \\
(44 \%)\end{array}$} & \multicolumn{2}{|c|}{$\begin{array}{c}\text { total }=7 \\
(22 \%)\end{array}$} \\
\hline
\end{tabular}

Fig. 4 - Répartition des familles en fonction de la provenance de leur terrain en 2012 (a. Les maris vivant sur la terre de leurs parents ont des sœurs; b. Familles ne possédant pas de droit à la terre et vivant dans la vila et/ou travaillant sur la terre des autres ; c. Familles dont l'occupation territoriale est jugée illégitime).

Lorsque la famille des deux conjoints possède un patrimoine foncier, le jeune couple expérimente la vie auprès de chacune des deux familles dans les premières années de résidence commune afin de déterminer dans quelle fratrie résidentielle il se fixera définitivement. Ce choix est absolument nécessaire et fondamental, puisqu'il détermine une fois pour toutes le lieu de vie et de travail du couple ainsi que l'unité politique à laquelle il s'intègre et avec laquelle il aura à « faire bloc ». Ce choix suppose aussi que des frères et sœurs ou des parents et leurs enfants pourront éventuellement s'affronter à l'avenir dans des conflits communautaires opposant plusieurs fratries résidentielles. En raison de conflits d'intérêts évidents, il est inconcevable de continuer à entretenir des relations de travail (localisation des jardins) et de résidence (localisation de la maison) dans deux fratries à la fois. Même lorsqu'un frère et une sœur appartenant à des fratries résidentielles différentes collaborent dans la réalisation de jardins « en société ${ }^{28}$ », le « maître » (dono) de la terre est toujours clairement identifié.

\section{D'UN TERRITOIRE EN RÉSEAU À UN SYSTÈME FONCIER D'UNITÉS TERRITORIALES}

La " communauté » est une réalité ambivalente. D’un côté, elle évoque une représentation idéelle d'un collectif d'individus moral et solidaire, de l'autre, elle 
consiste en une pratique de l'exercice du pouvoir dans l'ensemble des champs sociaux qui la composent (religieux, administratif, politique).

Dans les faits, la communauté est un espace de lutte pour le prestige, une sorte d'arène où chaque équipe (chaque fratrie résidentielle) positionne ses joueurs de façon stratégique afin d'accumuler du pouvoir. Nous nous sommes intéressés aux disputes les plus larvées, indicibles, qui remettent en cause les valeurs mêmes de la communauté idéelle, la raison d'être du groupe villageois. Nous avons cherché à déchiffrer les non-dits à partir de points de tension révélés par l'observation et les commentaires des habitants. L'utilisation de catégories de pensée locales (fratries résidentielles, communauté, etc.) nous a permis de décrypter des sens cachés dans ces discours, afin de comprendre de l'intérieur certains conflits réputés communautaires. Souvent présentés de manière dualiste (oppresseur capitaliste versus opprimé pauvre), ils soulèvent d'autres logiques inavouées (disputes intra-familiales sur la transmission du foncier, luttes pour le prestige). Les points de discorde évoqués n'amènent pas nécessairement à la violence physique. Les règles du vivre-ensemble entre parents et compères favorisent plutôt les relations sociales conviviales. Or, si la «communauté » constitue, en raison de ses valeurs intrinsèques, un système idéel cher à ses membres, sa nature même en fait l'axiome privilégié de la violence symbolique.

\section{Interpréter les conflits récents : la " communauté ", un concept en crise ?}

Ces dernières années, un nombre important de villages du bas Arapiuns connaît des conflits existentiels, en partie dus aux bouleversements institutionnels et territoriaux décrits ci-dessus. Avec la «communauté », les relations sociales entre les fratries résidentielles ont changé : le contrôle des institutions politicoreligieuses locales est exercé par une ou plusieurs fratries «fortes » dans un contexte de concurrence pour le prestige et le pouvoir. Même si, pour les institutions gouvernementales et un nombre croissant d'acteurs externes porteurs de projets, la «communauté » est devenue un interlocuteur politique de premier plan, par le biais de ses représentants ${ }^{29}$, c'est en réalité au sein des fratries résidentielles que sont encore aujourd'hui prises les décisions. Les fonctions de prestige étaient, et sont toujours, cumulées par les membres d'un groupe résidentiel dominant. En effet, l'ascension sociale de certaines fratries résidentielles leur a permis de jouer habilement de facteurs externes. Cette différenciation joua un rôle déterminant pendant les travaux de l'Incra.

À une époque où la majorité des chefs de famille de l'Arapiuns étaient analphabètes, le « dialogue » sur la législation nationale entre les « docteurs » de l'Incra et les membres de la communauté était en réalité une communication à sens unique et restait particulièrement hermétique pour ces derniers (difficulté encore dramatiquement observable aujourd'hui). Le président de la communauté, souvent originaire de l'extérieur ${ }^{30}$, faisait office de médiateur, voire de 
« traducteur », sans forcément comprendre mieux que les autres. Il accompagnait les techniciens dans leurs travaux de recensement et pouvait être amené à influencer la redistribution des terres dites « libres » (qui ne l'étaient en fait pas toujours) à de nouvelles familles. Le contrôle (et non l'exploitation) de ces terres était parfois revendiqué par des fratries résidentielles locales, sans que le dirigeant communautaire ne connaisse exactement les antécédents du lieu ou ne souhaite en tenir compte. Les récits sur les attributions de terres se terminent souvent par une phrase teintée d'amertume qui fait écho au problème structurel de la communauté : « Il [le président] se croyait le maître de tout. »

Certaines familles délimitèrent donc de grandes aires pour assurer leur reproduction sociale à moyen et long terme. Les grands perdants furent les cellules familiales atomisées souvent issues d'une migration récente ${ }^{31}$. Elles furent exclues de la redistribution, la plupart des terres étant déjà « occupées ». La vila-village, espace communautaire par excellence, devint le lieu de résidence permanent de ces familles sans terre. L'enregistrement au cadastre par l'Incra de cet espace représentait un défi de taille. Il était souvent construit sur les terres de la fratrie résidentielle qui en avait eu l'initiative. Si le président de la communauté en était membre, et c'était souvent le cas, il pouvait déclarer le territoire occupé par la vila en son nom propre. Dans certaines communautés étudiées, après le décès du fondateur, ses descendants entrèrent en conflit avec le reste des villageois afin de récupérer ces terrains (où avaient entre-temps été construites les principales infrastructures). Dans d'autres, on eut la clairvoyance d'ériger le village sur un territoire libre de toute occupation préalable. On abattit une portion de forêt pour y construire la salle communale et le terrain était enregistré au nom du saint patron des lieux.

La vila représente l'essence même de la communauté et la matérialise physiquement. Elle accueille l'ensemble des institutions : l'église, l'école, la salle communale, le terrain de football. Les disputes récentes de descendants réclamant la restitution des terrains familiaux sur lesquels avait été érigé le village sont aujourd'hui présentées par les actuels dirigeants communautaires (non issus du groupe du fondateur) comme le résultat d'un calcul individualiste. S'en suit un dialogue de sourds car deux logiques se télescopent : celle de la communauté idéelle pour les uns, celle de la fratrie résidentielle pour les autres.

La vila s'est imposée comme un espace emblématique et nécessaire au quotidien pour de nombreuses familles, qu'elles fassent ou non partie d'une fratrie résidentielle. La restitution du territoire occupé par la vila se solderait par la fin de l'existence de la communauté. Toutefois, du point de vue de la fratrie résidentielle du « maître » initial des lieux, il semblerait légitime d'être dédommagée pour le territoire cédé, puisqu'elle en a aliéné l'usufruit à la collectivité sans réussir à conserver, avec le temps, les fonctions de dirigeant (le charisme n'est pas héréditaire). Ainsi, si tous reconnaissent aujourd'hui le statut de primohabitante d'Uxícara à dona Vitória, personne ne dit explicitement que la vila de la 
communauté Vila Brasil fut construite sur ses terres et qu'au regard des pratiques locales, ses descendants y ont des « droits ». Sans doute doit-on comprendre cette situation comme un renversement de tendance : à l'époque, João Raymundo, commerçant éclairé, mit un espace et des infrastructures à la disposition de ses obligés (freguesia) pour les obliger davantage (dans une pure logique clientéliste). Quelques années plus tard, il eut encore soin de déclarer l'usufruit de ce patrimoine foncier à son nom, puis de réaliser l'ensemble des démarches dont il avait connaissance grâce à ses relations dans la ville de Santarém, jusqu'à en obtenir la pleine propriété et à en garantir la transmission légale à ses enfants. Il ne savait pas, cependant, que les descendants de ses obligés se mobiliseraient pour s'approprier un patrimoine idéel et symbolique qu'ils avaient tous contribué à forger et qu'ils considéraient désormais leur : l'idée d'une "communauté » solidaire et un ensemble de bénéfices incarnés dans la vila, principalement l'accès aux droits citoyens (école, église, infrastructures, affiliation administrative au syndicat, à la commune, inscription sur les listes électorales, etc.), mais aussi et surtout la participation à une forme de sociabilité « morale » qui est valorisée par opposition aux modes de vie des temps anciens où les riverains vivaient comme des « bêtes » dans la forêt.

\section{Conclusion}

Jusqu'à une période récente, le long de l'Arapiuns, l'occupation et la transmission de la terre étaient régies au sein de fratries résidentielles par des pratiques à tendance uxorilocale. Dans les années 1970, des facteurs politiques et institutionnels furent à l'origine de la formation de communautés engageant une réorganisation spatiale et politique. La fixation des familles dans des lieux précis fut finalement institutionnalisée à travers la régularisation foncière en lots individuels dans les années 1980 . Or, près de vingt ans après ces altérations dans la structure d'appropriation agro-mercantile, les mêmes familles furent encouragées par l'Incra à rendre tous les documents qu'elles détenaient (principalement des licences d'occupation, puisque très peu de riverains étaient devenus propriétaires de leur terre), qu'ils soient valides ou non, en raison de la création d'une nouvelle modalité territoriale : un Projet d'établissement agro-extractiviste (PAE), dont le titre foncier est collectif. Le PAE Lago Grande fut créé en 2005 et coïncide avec une conjoncture de désenclavement des communautés de l'Arapiuns et l'arrivée de nombreux projets (organisation politique, éducation, santé, économie familiale). En l'espace d'un siècle, la gleba Lago Grande da Franca est donc passée sous trois statuts fonciers différents : aucun statut spécifique jusqu'en 1970, puis délimitation d'une gleba fédérale où ont été enregistrées des occupations individuelles, et finalement, en 2005, une réserve écologique à statut collectif. Or, durant toute cette période, quel qu'ait été le statut de ce territoire, les 
pratiques de transmission de la terre au sein des fratries résidentielles a perduré avec quelques altérations dues à l'intervention sporadique d'agents externes.

Depuis la création du PAE Lago Grande, les acteurs externes - principalement les pouvoirs publics (préfecture de Santarém, etc.) et les ONG présentes dans la région - dialoguent avec les populations rurales par l'intermédiaire des associations communautaires ${ }^{32}$ créées pour l'occasion. En raison d'une catégorisation externe en termes de «communauté » ${ }^{33}$, le président et son groupe résidentiel sont amenés à «incarner» ladite communauté lors de ses transactions avec l'extérieur. Cette situation tend à transmettre pouvoir et prestige à une fratrie résidentielle en particulier, accentuant ainsi des différentiations sociales qui se retrouvent souvent à l'origine de conflits.

Mais qu'est-ce au juste que cette « communauté »? Figure institutionnelle encore floue, concept ambivalent, force est de constater que son fonctionnement apparent est un leurre. Dans la pratique, la logique sociale continue à être celle de la fratrie résidentielle, où sont prises les grandes décisions, quitte à le faire au nom de la collectivité. Car, si la communauté morale et idéelle est chère à tous, ne nous y trompons pas : lorsque l'intégrité de la fratrie est menacée, le premier réflexe est un repli sur le «centre » d'appartenance. Ce qui compte est la reproduction sociale de la fratrie résidentielle, même si elle doit changer plusieurs fois d'affiliation communautaire, voire même fonder sa propre communauté. L'identité est toujours corrélée à une parentèle et à un territoire, et non à la participation à une « communauté ».

Ainsi, la «communauté » est davantage une unité sociale situationnelle, déployée et invoquée lors de situations très spécifiques (où une intermédiation avec des acteurs externes est nécessaire) et contestée à d'autres moments (lorsque les intérêts de la fratrie résidentielle sont compromis). Nous retrouvons ici l'image de l'arène et des problématiques liées au factionnalisme local où des fratries résidentielles se disputent les fonctions honorifiques de représentation pour les prérogatives qu'elles confèrent, notamment le rôle d'intermédiation avec l'extérieur. Les laissés pour compte peuvent alors à leur tour se mobiliser afin de chercher une alternative à leur propre situation, se tournant eux aussi vers ces mêmes acteurs externes (État, ONG) qui en viennent à appuyer des groupes sociaux aux intérêts parfois divergents, favorisant l'émergence de contre-pouvoirs aux formes variées (Stoll et Folhes 2013) ${ }^{34}$. Dans un même village, on peut trouver des lieux de culte concurrents, des mouvements sociaux aux objectifs antagonistes, des revendications ethniques, etc. Il devient évident que certaines de ces situations sont accentuées par les projets assistentialistes qui permettent de creuser l'écart différentiel entre groupes sociaux de condition initialement similaire.

Or, pour de nombreux acteurs externes, la communauté reste cet idéal de familles unies autour d'un projet de vie commun. Cette illusion communautaire entretient la rhétorique collectiviste et les symboles qui lui sont attachés. 
Pourtant, la mise en œuvre de politiques publiques (ou de projets d'assistance) dans les communautés rurales amazoniennes ne parvient pas toujours à instaurer des droits collectifs ou à redistribuer démocratiquement et de façon égalitaire les bénéfices entre les habitants. Elle tend au contraire à accroître les conflits factionnels au sein du village et à provoquer une certaine désillusion chez les habitants. Bien entendu, ceci est en totale contradiction avec la conception qu'en ont les institutions publiques et autres acteurs externes.*

* Manuscrit reçu en juin 2013, accepté pour publication en décembre 2013.

\section{Notes}

Patrick Menget, Chantal Medaets, Véronique Boyer, Isabelle Daillant et deux relecteurs anonymes ont, par leur lecture attentive et leurs suggestions, grandement contribué à l'élaboration de cet article. Nous les remercions chaleureusement.

1. Ces travaux ont été financés par le programme GEOMA « Modelagem de arranjos institucionais em múltiplas escalas - Model AGER/NÓMOS »(CNPq/MCT-INPE), coordonné par Roberto Araújo. Des aides ponctuelles du REFEB et de la région Île-de-France ont pris en charge une partie des frais de mobilité. Nous les remercions. L'enquête ethnographique, réalisée entre 2008 et 2012, a alterné des périodes de terrain de courte durée (1 à 3 semaines) dans dix communautés et un séjour prolongé de 13 mois dans deux communautés. Nous avons travaillé à partir des récits de recomposition mémorielle des habitants actuels ainsi que de nos propres observations.

2. Le décret $6.040 \mathrm{du} 7$ février 2007 institue la politique nationale de développement durable des peuples et communautés traditionnelles et les définit comme des « groupes culturellement différenciés et se reconnaissant comme tels, qui possèdent des formes d'organisation sociale qui leur sont propres, qui occupent et utilisent des territoires et des ressources naturelles comme condition de leur reproduction culturelle, sociale, religieuse, ancestrale et économique, en utilisant les savoirs, les innovations et les pratiques générés et transmis par la tradition » (art. 3-I). Les traductions des citations ont été réalisées par nos soins.

3. Dans l'écosystème de terre ferme (qui caractérise les rives de l'Arapiuns et du Tapajós), comme dans celui de várzea (localisé le long du lit de l'Amazone), une partie importante des terres est immergée pendant la saison des pluies. La différence réside dans la nature du sol lors de l'étiage. Dans la várzea, il laisse apparaître des terres arables très fertiles et de grasses pâtures ; dans la terre ferme, des plages de sable et une végétation arbustive.

4. Il s'agit d'individus exerçant une forme de pouvoir de domination local fondé sur un endettement plus symbolique que réel (aviamento), souvent camouflé par des relations de compérage et un paternalisme latent. Sur ce thème, se reporter aux travaux de Geffray (1995), Léna (1996) et Araújo (1993).

5. Période où, profitant de la fin de la montée des eaux (vers juillet), les poissons remontent la rivière par bancs afin d'aller se reproduire en amont, où les chances de survie sont les meilleures.

6. Pendant la réalisation des enquêtes de terrain, l'électricité a été installée dans plusieurs communautés.

7. Quelques localités davantage peuplées avaient adopté l'appellation de vila sans constituer pour autant une unité administrative.

8. Soulignons la différence avec ce qu'a relevé Kohler (2009) chez les Pataxó, où « "autrefois" [...] les fêtes de saints étaient organisées par la communauté entière ». Dans l'Arapiuns, jusqu'à une période récente, les statuettes de saints étaient détenues par des individus spécifiques, considérés comme étant leurs « propriétaires » (donos). Ceux-ci étaient souvent les initiateurs et les hôtes de la fête patronale, qui se déroulait dans un espace mis à la disposition des convives. Ce n'est qu'après la formation des 
« communautés » que certains fondateurs de villages «donnèrent » leur statuette à la communauté villageoise et qu'il incomba désormais à la coordination de catéchèse du village d'organiser les festivités.

9. Ainsi, l'église d'Arapixuna fut construite dès 1875 par un propriétaire terrien ; à Vila Socorro, la chapelle date de 1935 ; et un commerçant local offrit la statue de la vierge à l'église de Curuai en 1900 (Diocèse de Santarém 2006).

10. En 1755, le marquis de Pombal réorganise administrativement l'Amazonie.

11. Terres concédées à des tiers par la Couronne portugaise pendant la période coloniale pour encourager l'occupation territoriale et le développement de l'agriculture, de l'élevage et de l'extractivisme.

12. À titre d'exemple, en 1909, sur 1572 déclarations d'occupation (declarações de posse) enregistrées à Santarém, quatre seulement concernaient des terres situées le long de l'Arapiuns, toutes les autres étant dans la várzea (Muniz 1909). Il ne s'agissait donc pas d'une pratique courante.

13. Tous les noms de personnes utilisés dans cet article sont fictifs.

14. Végétation arborée composée principalement de palmiers tucumã (Astrocaryum aculeatum).

15. Les franciscains s'installèrent à Vila Curuai en 1963.

16. En 1984, le diocèse de Santarém est réorganisé en « régions pastorales 》 afin de rendre son action missionnaire plus efficace. Les communautés de l'Arapiuns passent alors dans la circonscription des Verbitas.

17. À Santarém, ce syndicat (le STTR) envoyait des militants sac au dos accomplir des « visites de la base » dans les communautés.

18. Maison rustique construite avec des matériaux de la forêt disponibles sur place. Cette habitation, où l'on passe pourtant le plus de temps, n'est pas appelée « maison », à l'inverse de celle du village, où l'on ne passe que le week-end.

19. Comprendre ici déboisé/boisé : la forêt est un espace sauvage. Il faut donc la domestiquer (amansar) en la coupant.

20. La possession se différencie de la notion juridique de propriété. La première est, en principe, inaliénable. Dans la pratique, les posseiros - comme on appelle les occupants de terres fédérales sans titre de propriété - réalisent des transactions de vente, parfois de bonne foi, ce qui contribue à la complexité de la situation foncière locale.

21. Date du coup d'état militaire et de l'adoption du nouveau Statut de la terre.

22. Une gleba est une aire territoriale continue qui est enregistrée au cadastre au nom de l'État fédéral sous un titre foncier unique. Elle peut avoir différentes destinations (terre indigène, assentamento, colonisation privée...).

23. Le cas des familles sans terre de l'Arapiuns (terre ferme) est différent de ce qui a pu être décrit pour la várzea (Futemma 2006). Sur la terre ferme, le mode d'occupation spécifique du territoire en fratries résidentielles explique la présence de familles atomisées sans terres. Celles-ci ont perdu leur droit à la terre suite à une exclusion ou après une alliance matrimoniale peu stratégique (avec un conjoint sans terre par exemple). Dans la várzea, il semble que l'espace physique restreint soit à l'origine du manque de terre, puisque la contiguïté des lots ne permet pas aux descendants mariés de rester sur le terrain des parents.

24. Licences qui pouvaient éventuellement, au bout de quatre ans et contre le paiement d'une certaine somme, ouvrir droit à un titre de propriété définitif.

25. Le recensement économique de 1920 ne prend en compte que les exploitations qui réalisent un chiffre d'affaire supérieur à 500000 réis, c'est-à-dire qu'en est exclue l'agriculture vivrière.

26. Cette pratique, assez fréquente, consiste en l'adoption, par une grand-mère, d'un ou plusieurs de ses petits-enfants afin qu'ils soient élevés comme s'il s'agissait de ses propres enfants.

27. Les belles-filles sont souvent suspectées d'entretenir des liaisons extraconjugales.

28. Les jardins «en société » sont exploités par deux personnes à la fois : l'un fournit la terre et l'autre la travaille. La production est ensuite divisée en deux parts égales. 
29. Le président de la communauté (ou de l'assemblée communautaire), le délégué syndical, le coordinateur de la catéchèse, qui sont souvent tous issus d'une même fratrie résidentielle.

30. Qu'il soit l'un de ces commerçants charismatiques, ou bien qu'il ait intégré le village suite à une union uxorilocale avec une femme locale.

31. Les familles migrantes isolées de leur fratrie résidentielle d'origine sont toujours suspectes. Chacun sait qu'une migration n'est jamais volontaire et résulte d'un conflit, d'une instabilité liée à l'absence de terre propre, ou encore d'une fuite suite à une accusation de sorcellerie ou d'un acte contraire aux mœurs.

32. Les communautés ont été incitées à créer et enregistrer légalement des associations communautaires qui sont en charge de recevoir et d'administrer les bénéfices (matériels, monétaires) issus des projets. La figure du président de la communauté est alors remplacée par celle du président de l'association.

33. Cette catégorisation s'opère au nom d'une image idéalisée d'organisation sociale communautaire qui permettrait la redistribution équitable entre ses membres de ressources limitées.

34. Boyer (2008) a montré comment, dans les villages riverains, l'ouverture de lieux de culte concurrents (catholiques versus évangéliques, etc.) était une façon d'investir le champ religieux et moral pour mettre en place des contre-pouvoirs dans les luttes factionnelles locales.

\section{RÉFÉRENCES CITÉES}

Adams Cristina, Rui Murrieta, Walter Neves (éd.)

2006 Sociedades caboclas amazônicas: modernidade e invisibilidade, Fapesp/ Annablume, Sao Paulo.

ARAúso Roberto

1993 La Cité domestique : stratégies familiales et imaginaire social sur un front de colonisation en Amazonie brésilienne, thèse de doctorat, ethnologie, université Paris X-Nanterre.

2009 «De la "communauté" aux "populations traditionnelles" ", Nuevo Mundo/Mundos Nuevos [en ligne], Colloques, mis en ligne le 6 juillet 2009, consulté le 23 décembre 2014 [http://nuevomundo.revues.org/56593].

AraúJo Roberto, Philippe LÉnA

2010 "Da predação à sustentabilidade na Amazônia: a difícil metamorfose », in Ph. Léna, R. Araújo (éd.), Desenvolvimento sustentável e sociedades na Amazônia, Museu Paraense Emílio Goeldi, Belem, pp. 13-55.

Arquivo PÚblico do Estado do Pará (APEP)

1837 Fonds du Secrétariat de la présidence de la Province (FJSSA), codex 888, document 123.

2009 Catalogue des sesmarias.

BARriga Letícia Pereira

2007 Ecuipiranga: o berço revolucionário no baixo Amazonas (1835-1837), trabalho de conclusão de curso, Universidade federal do Pará, Belem.

Borges Luiz

1994 «O nheengatú na construção de uma identidade amazônica », Boletim do Museu Paraense Emílio Goeldi, série « Antropologia », 10 (2), pp. 107-135. 
BOURDIEU Pierre

1980 Le Sens pratique, Les Éditions de Minuit, Paris.

BOYER Véronique

2008 La Renaissance des perdants: expansion évangélique et migrations en Amazonie brésilienne, Institut de recherche pour le développement/ Karthala, Paris.

DiocÈsE De SANTARÉM

2006 Anuário da Diocese de Santarém, Gráfica e Editora Tiagão, Santarém.

FAUlHaber Pricila

1986 "Tradition et changement dans le moyen Solimões : structure sociale et mouvements de revendication à base territoriale ", Cahiers des sciences humaines, 22 (3-4), pp. 389-400.

Futemma Célia R. T.

2006 «Uso e acesso aos recursos florestais: os caboclos do baixo-Amazonas e seus atributos sócio-culturais ", in C. Adams, R. Murrieta, W. A. Neves (éd.), Sociedades caboclas amazônicas: modernidade e invisibilidade, Fapesp/Annablume, Sao Paulo, pp. 237-260.

GALVÃo Eduardo

1976 Santos e visagens: um estudo da vida religiosa de Itá, Baixo Amazonas, Companhia Editôra Nacional, Sao Paulo [1955].

GeFFray Christian

1995 Chroniques de la servitude en Amazonie brésilienne, Karthala, Paris.

GoODY Jack

1975 «The fission of domestic groups among the Lodagaba », in J. Goody (éd.), The developmental cycle in domestic groups, Cambridge University Press, Cambridge, [1962], pp. 53-91.

Gow Peter

1991 Of mixed blood: kinship and history in Peruvian Amazonia, Clarendon Press, Oxford.

Gross Daniel R.

1973 « Factionalism and local level politics in rural Brazil », Journal of anthropological research, 29 (2), pp. 123-144.

HARRIS Mark

2000 Life on the Amazon: the anthropology of a Brazilian peasant village, Oxford University Press, New York.

2004 "Peasants on the floodplains: some elements of the "agrarian question" in riverine Amazonia ", in S. Nugent, M. Harris (éd.), Some other Amazonians: perspectives on modern Amazonia, Institute for the study of the Americas, Londres, pp. 81-103.

2010 Rebellion on the Amazon: the Cabanagem, race and popular culture in the north of Brazil, 1798-1840, Cambridge University Press, New York. 
Houtzager Peter

2004 Os últimos cidadãos: conflito e modernização no Brasil rural (1964-1995), Globo, Sao Paulo.

IORIS Edviges

2009 «Identidades negadas, identidades construídas: processos identitários e conflitos territoriais na Amazônia », ILHA Revista de Antropologia, 11 (1-2), pp. 220-264.

KoHLER Florent

2009 «Commentaire sur "De la 'communauté' aux 'populations traditionnelles' : aspects de la modernité amazonienne" (Roberto Araújo) », Nuevo Mundo/Mundos Nuevos [en ligne], Colloques, mis en ligne le 07 juillet 2009, consulté le 23 décembre 2014 [http://nuevomundo.revues.org/56678].

LÉNA Philippe

1996 «Les rapports de dépendance personnelle au Brésil : permanences et transformations ", Lusotopie, 1996, pp. 111-122.

LEROY Jean-Pierre

1991 Uma chama na Amazônia, Editora Vozes, Rio de Janeiro.

Lima Deborah DE MaLgalHães

2004 "The roça legacy" land use and kinship dynamics in Nogueira, an Amazonian comunity of the Middle Solimões region ", in S. Nugent, M. Harris (éd.), Some other Amazonians: perspectives on modern Amazonia, Institute for the study of the Americas, Londres, pp. 12-36.

Menget Patrick

1985 « Jalons pour une étude comparative », Journal de la Société des américanistes, 71, pp. 131-141.

Muniz João de PALma

1909 Índice dos títulos de terras expedidos pelo governo do estado na Administração do Exm. Snr. Dr. Augusto Montenegro: 1901-1908, Secretária de Estado de Obras Públicas, Belem.

Nugent Stephen

1993 Amazonian caboclo society: an essay on invisibility and peasant economy, Berg Publishers (Explorations in anthropology), Oxford.

O'DWYer Eliane CANTARINo

2005 "A construção da várzea como problema social na região do baixo Amazonas », in D. Lima (éd.), Diversidade socioambiental nas várzeas dos rios Amazonas e Solimões, Ibama/ProVárzea, Manaos, pp. 207-264.

PRelazia de Santarém

1970-1983 Anuário, 7-12, 14-17, s. n., Gráfica e Editora Tiagão, Santarém.

REPÚBLICA DOS ESTADOS FEDERADOS DO BRASIL

1927 Recenseamento do Brazil, Realizado em 1 de setembro de 1920, Relação dos proprietarios dos estabelecimentos ruraes recenseados no Estado do Pará, 
Ministerio da Agricultura, Industria e Commercio, Directoria Geral de Estatistica, Typ. da Estatistica, Rio de Janeiro.

\section{SiLva Ligia Maria OsóRIO}

2008 Terras devolutas e latifúndio, Editora da Unicamp, Campinas.

STOLL Émilie

2014 Rivalités riveraines: territoires, stratégies familiales et sorcellerie en Amazonie brésilienne, thèse de doctorat, anthropologie, École pratique des hautes études, Paris/Universidade Federal do Pará, Belem.

Stoll Émilie, Ricardo TheOPHILO FolHes

2013 «Frères ennemis : la participation à l'épreuve des factions en Amazonie brésilienne », Cahiers des Amériques latines, 72-73, pp. 141-160. 
